\title{
Las mujeres universitarias embarazadas y el factor que incide en su avance y el rendimiento académico
}

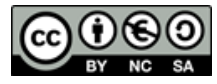

Pregnant universitary women and the factor that influences their progress and academic performance

Efraín Velasteguí López. ${ }^{1}$

Recibido: 05-09-2018 / Revisado: 15-09-2018 / Aceptado: 23-09-2018 / Publicado: 05-10-2018

Resumen.

DOI: https://doi.org/10.33262/concienciadigital.v1i4.905

El embarazo adolescente en ecuador es un mayor porcentaje donde el instituto ecuatoriano de estadística y censos muestra en el año $201443.3 \%$ de mujeres embarazadas tomando en cuenta que son menores de 15 años y el 3\% señoritas de 17 años y mamas de 18 a 19 años y según los informes de cada $43 \%$ adolescentes de 12 a 19 años son madres. La planificación familiar permite a las parejas tener una mayor oportunidad para la reflexión sobre sus hijos ya que facilitara un mejor desarrollo de la economía ya que la planificación familiar es parte del esfuerzo se supervivencia infantil por existen casos que algunos adolescentes provocan el embarazo de temprana edad o algunos llegan hijos a cada año o hijos no deseados están al propenso de abandono o descuido que puede llegar al aborto. El nuevo estudio de estado anídense afirma promover el acceso gratuito de anticonceptivos y otros medicamentos para combatir el embarazo y acudir con las clases de educación sexual para prestar un mejor conocimiento sobre el sexo aunque los anticonceptivos no son muy seguros para combatir el embarazo el implante cuenta con más seguridad y prevenir el embarazo adolescente.

El embarazo es un problema de salud como las universidades suelen caerse y cada vez más universitarias de cada región suelen embarazarse sin embargo es una problemática que trae dificultades en el rendimiento académico. Este estudio es basado en una realidad de las madres y padres solteros de las universidades que merecen seguir el

\footnotetext{
${ }^{1}$ Ciencia digital, Ambato, Ecuador, luisefrainvelastegui@cienciadigital.org
} 
estudio en el ecuador ya que plantea la creación de guarderías universitarias para que las madres puedan graduarse si ningún problema debido al embarazo.

Palabras claves: Mujer Embarazada, Charlas Planificación familiar.

\section{Abstract.}

Adolescent pregnancy in Ecuador is a higher percentage where the Ecuadorian Institute of statistics and censuses shows in $201443.3 \%$ of pregnant women taking into account that they are under 15 years old and 3\% young ladies of 17 years old and breasts of 18 to 19 years and according to the reports of every $43 \%$ adolescents from 12 to 19 years old are mothers. Family planning allows couples to have a greater opportunity to reflect on their children and to facilitate a better development of the economy since family planning is part of the child survival effort because there are cases that some adolescents cause early pregnancy or some children arrive every year or unwanted children are prone to abandonment or neglect that can reach abortion. The new state study encourages promote free access to contraceptives and other drugs to combat pregnancy and go with sex education classes to provide better knowledge about sex although contraceptives are not very safe to combat pregnancy the implant account more safely and prevent teenage pregnancy.

Pregnancy is a health problem as universities tend to fall and more and more universities in each region tend to get pregnant, however it is a problem that brings difficulties in academic performance. This study is based on a reality of the single mothers and fathers of the universities who deserve to follow the study in the equator since it raises the creation of university nurseries so that mothers can graduate if no problem due to pregnancy.

Keywords: Pregnant Woman, Talk family planning.

\section{Introducción.}

La presente investigación de la situación de las mujeres embarazadas pretende acercarse a la historia y realidad de la identidad femenina y la maternidad y en esta información se llevara a cabo en dar a conocer el significado de ser madre de las mujeres solteras o con compromiso con hijos por lo cual son afectadas en el estudio académico por falta económicas y falta de tiempo y apoyo, donde hay muchas mujeres con hijos que se dedican al estudio buscando una meta más que cumplir para que en el trascurso del año tenga su vida profesional y por ello no tienen un lugar adecuado donde dejar a sus hijos.

De la misma manera en este informe se pretende dar solución a esta problemática con las autoridades que serán elegidas en el año 2019 en la cual se estima solicitar la creación de guardería universitaria y así también dictar o efectuar constantemente de charlas sobre 
planificación familiar ya sea capacitados por autoridades y estudiantes de la institución de educación superior con la cual se pretende reducir el índice de mujeres embarazadas a temprana edad en la Universidad Técnica de Cotopaxi extensión La Maná para dar un mejor servicio a las madres en busca de un sueño por cumplir para su vida y presenten un mejor rendimiento en el centro académico en el transcurso del año hasta finalizarlo y obtengan su título sin ningún problema, y en la presente investigación el plan de ayuda de madres universitarias con hijos que obtengan un mejor rendimiento académico .

Figura N 1. Mujeres embarazadas
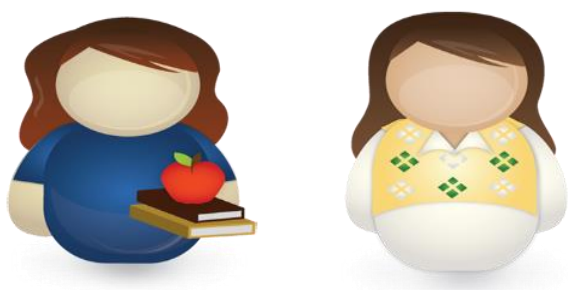

Fuente: Elaboración propia.

\section{Desarrollo:}

El autor (Garcia Andres, 2018) menciona que unas de las tareas pendientes, de cara a la conmemoración del día de la madre es la reducción del índice de embarazo adolecentes en Ecuador el informe de mujeres ecuatorianas dos década de cambio 1995-2015 recoge datos sobre fecundidad en jóvenes y su incidencia en el país, basados en datos del instituto ecuatoriano de estadísticas y censos (INEC), el documento muestra que, en el 2014, hay de 49,3 de cada 100 hijos nacidos vivos y fueron de madres adolescentes, de hasta 19 años si se toma en cuenta los rangos de edad, las progenitoras menores de 15 años, representan el 3\% y las madres entre 15 y 17 años conforman el $23 \%$ mientras que las mamas de entre 18 y 19 años ocuparon el $23.3 \%$ segun el informe, en total 12 de cada 100 adolescentes de 12 a 19 años son madres.(Garcia Andres, 2018)

Tabla N 1: Porcentaje de mujeres Embarazadas a temprana edad

\begin{tabular}{|c|c|c|}
\hline Progenitoras menores & 15 años & $3 \%$ \\
\hline Madres & $15 y 17$ años & $23.3 \%$ \\
\hline Parto adolescente & $10 y 14$ años & $78 \%$ \\
\hline Embarazos de menores & A 20 años & $15 \%$ \\
\hline
\end{tabular}

Fuente:(Garcia Andres, porcentage de mujeres embarazadas , 2018)

Análisis: Es una cantidad muy alta de mujeres embarazadas en nuestro país que mullos de ellos son menores de edad esto sucede por falta de charlas académicas es decir presentar un 
poco de conocimiento sobre la sexualidad en cada institución principalmente en las escuelas y colegios porque ellos son los más principales que salen afectados en esto del embarazo por falta de conocimiento en no saber cómo cuidarse lo mismo deberíamos hacer con los padres en hacer una reunión y mencionar sobre la sexualidad para que así pueda contar con sus hijas y no descuidarlos así existirán menos adolescentes que sufran con el embarazo y sepan ser responsables y cuidarse de sí mismo y en estos casos hoy en día el porcentaje de mujeres embarazadas debe ser más alto como son afectadas desde el colegio hasta la universidad jovencitas a temprana edad con hijos y estudiando donde generar un problema para ellas por que no tienen dónde acudir a sus hijos algunas madres lo llevan junto a ellos donde puede ser molestoso para algunos docentes algunas contienen a su pareja algunas son madres solteras y no todos tienen el apoyo familiar algunas tendrán problemas de esto y no podrán terminar el estudio y se retiraran y buscaran la manera de dar lo mejor para sus hijos y no podrán cumplir sus propósitos que lo llevan van a cabo.

Figura N 2. Mujeres Embarazadas

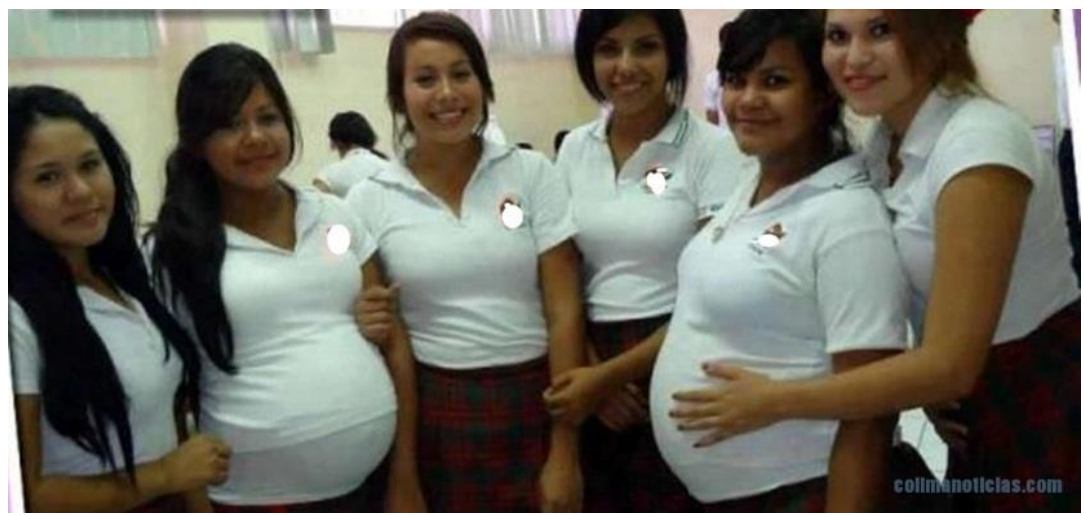

Fuente:(Noticias Redaccion Colima, 2015)

\section{Charlas sobre la planificación familiar}

La Br. (Maradiaga Cuadra Yamilia ,Weimar Flores Sherlie, 2007)nos informa que la planificación familiar permite a las parejas tener la oportunidad para reflexionar adecuadamente sobre el significado de los hijos, sobre sus necesidades y sobre efectos que tiene la llegada de un nuevo ser para, los padres mismos, para los otros hijos si los hay y para la comunidad en general y es una condición que facilita el desarrollo de socio económico y mejorar la economía ya que la planificación familiar forma parte del esfuerzo para mejorar la supervivencia infantil ya que las vidas de millones de niños podrían salvarse mediante la prevención de embarazos no deseados y de aquellos otros que ocurren demasiado temprano demasiado tarde o muy seguido los niños no deseados están más propensos al abandono y al descuido. Algunos investigadores sostienen que la existencia de una necesidad insatisfecha se debe principalmente a la falta de acceso a los servicios de anticonceptivos otros aseguran que entre las causas de la necesidad insatisfecha se incluye la falta de conocimiento el temor 
a los afectos secundarios y la desaprobación social y familiar. Factores que podrían intervenir en la insatisfacción de necesidades en planificación familiar:

Figura N 3. Instalaciones de nesesidades

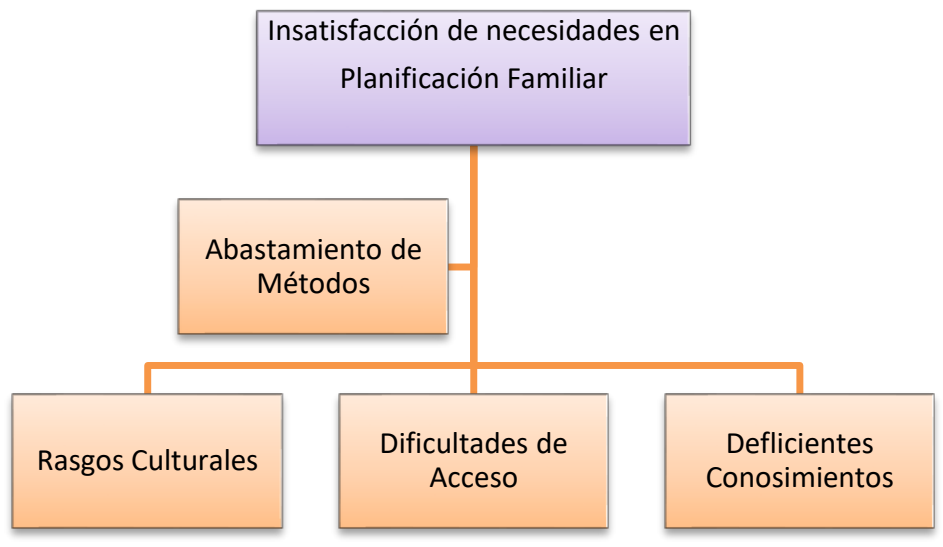

Fuente: Elaboración propia.

Análisis: Ecuador es el tercer país en la región con la tasa más alta que existen mujeres embarazas y no todas las mujeres desean tenerlo o no pueden desarrollarse y van presentándose problemas diferentes y por ello que algunas madres reflexionaran y lo tendrán a su bebe y otras buscaran la manera en deshacerlos con el aborto en esto cuenta de mucho peligro que puede llegar hasta la muerte y esto pasa por la inresposablidad de cada uno muchos adolescentes busca al aborto por el miedo de sus padres o su vez llegan a tener pero se presentan problemas que sus bebes llegan hacer prematuros o tienen discapacidades diferentes y presentan diferentes problemas por los casos de inseguridad y cuidado de la madre así como no todas las madres llegan hacerse de compromiso y llegan hacerse madres soltera que por ello existen bebes no deseados y en este plan algunas familias sacaran adelante a sus hijos junto a ellos pero esto cera un esfuerzo de ellos y algunos se preocupara en dar lo mejor para sus hijos y ello buscaran u mejor trabajo donde llegue suficiente dinero a la casa en caso de mujer soltera será al mismo tiempo padre y madre para sus hijos y eso sería lo más complicado para una madre responsable en este caso presentamos el porcentaje de mujeres que utilizan métodos anticonceptivos.

Tabla N 2. Porcentaje de Protección para evitar el Embarazo

\begin{tabular}{|c|c|}
\hline Métodos de anticonceptivos & $\%$ \\
\hline Ninguna escolaridad $(\mathrm{n}=62)$ & $61.3 \%$ \\
\hline Primaria $(\mathrm{n}=1.38)$ & $82.6 \%$ \\
\hline Secundaria o universitaria $(\mathrm{n}=18)$ & $88.9 \%$ \\
\hline
\end{tabular}

Fuente: (Yamilia, 2007) 


\section{Reducir el índice de mujeres embarazadas a temprana edad}

La (Universia, 2014) nos da la información que un nuevo estudio estadounidense afirma que promoviendo el acceso gratuito a anticonceptivos y la educación sexual es posible reducir las posibilidades de embarazo y posteriores decisiones de aborto como el embarazo adolescente es un fenómeno global que despierta enorme preocupación entre las autoridades nacionales e internacionales sin embargo un estudio público en el new england jounarl of medicine las mujeres deberían seguir educación sexual y tienen al acceso gratuito a anticonceptivo corren menos riesgo de embarazo o de decidir abortar el estudio promueve especialmente los métodos de anticonceptivos de acción prolongada como los dispositivos o los implantes ya que son eficaces para combatir los embarazos adolescentes. Las adolescentes pueden tomar buenas decisiones cuando están informadas y tienen el apoyo de sus familiares u otros adultos de confianza.

Figura N 4. Métodos de protección

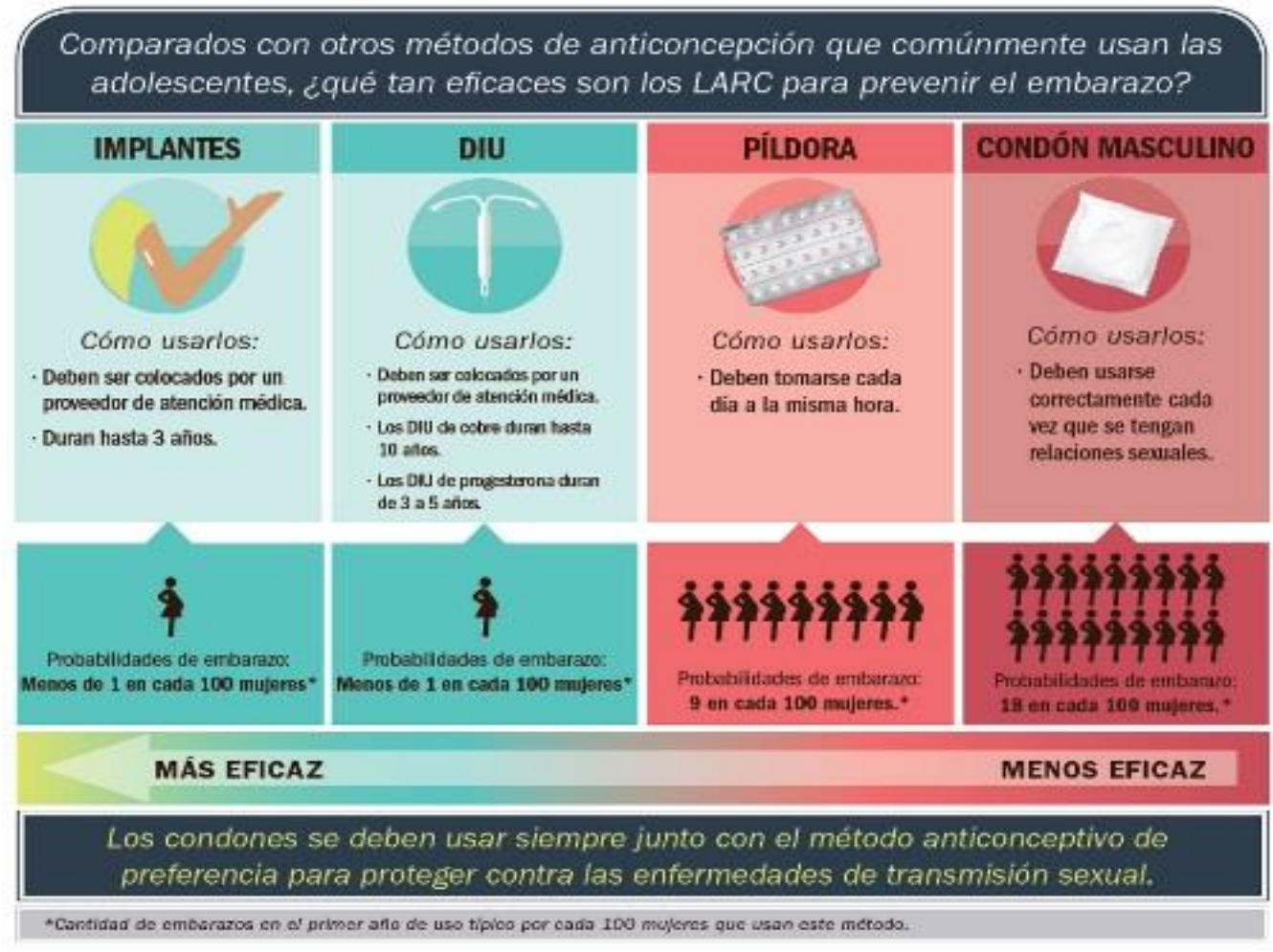

Fuente: (CDC, 2015)

\section{Análisis:}

En nuestro país deberíamos hacer lo mismo para que así vayan disminuyendo el porcentaje del embarazo en mujeres adolescentes deberíamos compartir conocimientos sobre la sexualidad y en cada farmacia den el acceso gratuito a los anticonceptivos y a los 
preservativos en caso de los jóvenes hay diferentes formas de cuidarse y en ese transcurso también ayudar a los adolescentes con charlas respecto al sexo que es algo normal en una relación o a su vez algunos lo toman por placer o pasatiempo y eso puede llegar hasta un cierto punto donde pueden quedarse embarazadas y otro tipos de los adolescentes no lo toman a bien no se acercan a las farmacias por la vergüenza y eso hay que darles entender que no es algo de otro mundo que es mejor que se cuiden para prevenir el embarazo, pero en caso que el adolecente ya se encuentra embarazada no se debe utilizar ningún medicamento porque esto puede causar el aborto o a su vez puede presentarse un problema grave hacia la madre debido a estos casos presentar charlas sobre la sexualidad

\section{Creación de guarderías universitarias}

El señor (ORTIZ ASAEL, 2014) menciona que el embarazo en adolescentes es un problema de salud de acuerdo con la organización mundial de la salud (OMS), las universitarias suelen caerse, no corren ese riesgo; sin embargo, los datos demuestran que cada vez más universitarias de distintas regiones del mundo, sobre todo Latinoamérica, se embarazan, lo que pone en tela de juicio la calidad de la educación que reciben en las universidades. El embarazo durante la etapa universitaria es una problemática que trae problemas en lo social familiar y en general en su rendimiento académico.

\section{Análisis:}

En este caso de mujeres embarazadas universitarias es una situación muy complicada por lo que deben dar tiempo tanto a su hijo como al estudio pero para algunas madres se les complica mucho y tienen su rendimiento académico muy bajo porque no cuentan con la facilidad del tiempo y no tienen donde dejar a sus hijos en caso de mi cantón La Maná suelen pasarlo existen muchas madres de la Universidad Técnica de Cotopaxi estudiando algunas suelen llevarlos a sus bebes a la institución y algunos suelen dejarlo con sus familiares y de la preocupación de los hijos no salen asistir las horas completas como es debido y de esa forma se presentan los problemas sobre el rendimiento académico que algunas madres suelen arrastres algunas materias y debido a esto toman la decisión en retirarse de la universidad y no pueden cumplir su vida profesional y también hay casos que llegan a retirarse por falta de la economía o falta de estudios que muchos trabajan para estudiar y eso es un problema muy grande esto también suelen pasar a muchas señoritas y jóvenes estudiantes que ellos buscaran la manera de seguir adelante con los estudios y mientras haya esa oportunidad lo aprovecharan . 
Figura N 5. Graduación Universitaria

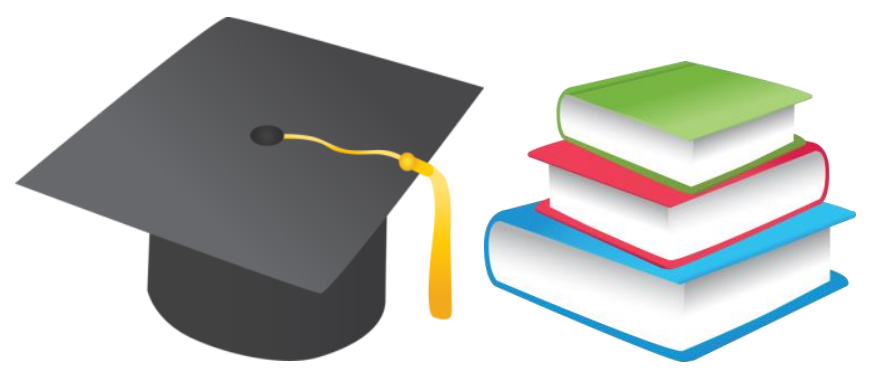

Fuente: Elaboración propia.

Los señores (Fajardo Zila , Figeroa Erica,Garces Norma,, 2016)Menciona que este estudio es un análisis de la realidad de las madres y padres solteros de las universidades y que merecen seguir sus estudios universitarios en el ecuador ya que plantean la creación de guarderías universitarias para que los hijos de los estudiantes que acuden al estudio y se dé la oportunidad de graduarse.

\section{Análisis:}

Hablando sobre la necesidad de mi prestigioso cantón La Maná esto cuenta de la realidad de muchos adolescentes o adultas con hijos acuden a la universidad técnica de Cotopaxi y para ello se debe mencionar el tema con las autoridades de la universidad para que ellos hagan un oficio con las autoridades que pertenecen al gobierno que nos ayuden con una guardería universitaria para que así las madres tengan donde dejar a sus hijos y puedan mejorar su rendimiento académico y puedan estar tranquilas sabiendo que están en un lugar seguro donde a la hora de salida podrán retirarlos con calma y esto se daría un paso más para sacar adelante a la universidad por que presentarían la mejor oportunidad para las madres que quieren estudiar y ser algo en la vida y el proceso de aprendizaje sería mejor .Como en Quito, Guayaquil, Tulcán, ya existen guarderías que favorezcan a los estudiantes con hijos a terminar sus estudios si hay en esas ciudades porque no luchamos para que cuente con una guardería en nuestra universidad si muchas madres lo necesitan y para ellas darían un mejor agradecimiento por prestarles una oportunidad más en sus vidas. 
Figura N 6 .Guardería Universitaria

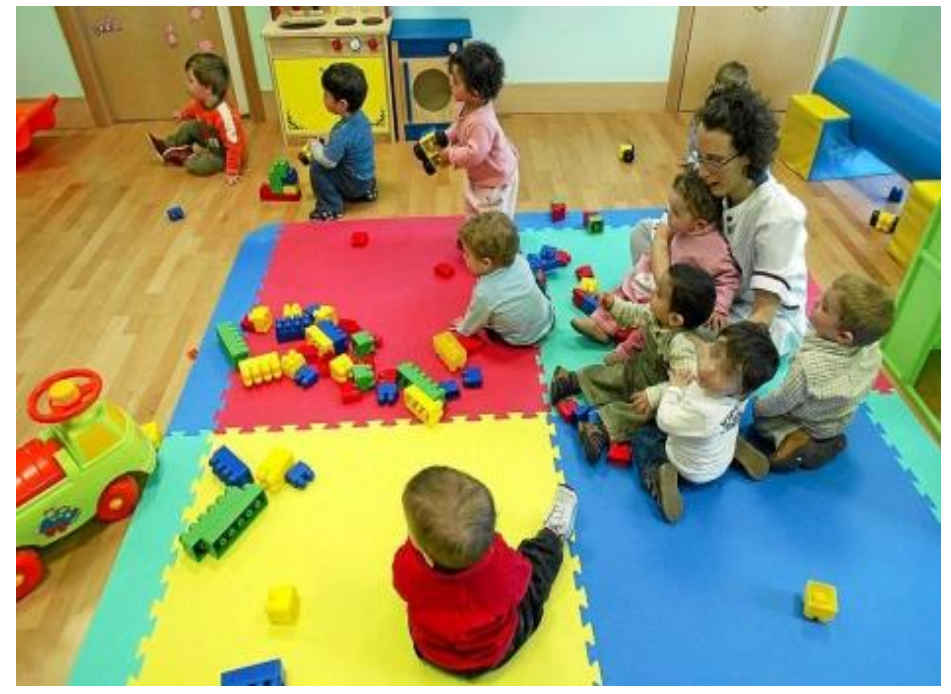

Fuente: (Guarderia, 2012)

\section{Conclusiones}

- Concluyo que el embarazo adolescente es mayor porcentaje en todo do Latinoamérica por lo tanto en uno de ellos esta nuestro país que presenta un alto nivel de mujeres embarazadas a temprana edad por el descuido de uno mismo y de los padres o falta de conocimiento, esto presenta un problema a nuestro país de ver adolescente de 12 a 18 años embarazadas o de compromiso donde pierden la oportunidad de seguir estudiando algunas madres no terminan ni el colegio o algunas lo aran por el apoyo que sustenta su familia o pareja pero no es fácil tomar el transcurso del estudio con hijo porque un hijo necesita de mucha atención y protección de su madre.

- Las implicaciones del embarazo en la adolescencia resultan un impacto negativo como tanto para hombres y mujeres, con manifestaciones en el ámbito familiar estudio y tiempo que registra un problema a su rendimiento académico la cual se busca una solución para no afectar más a los adolescentes en el transcurso académico.

- De acuerdo a la investigación presentada se concluye que existe una solución de como disminuir el embarazo en la adolescencia ya que es un tema muy importante para el bien de los adolescentes tomados en cuenta que se presenta un mayor problema dentro del ámbito personal o familiar.

- Los resultados de la investigación cuentan con una guardería universitaria tomando encuentra con las autoridades de la institución como los de gobierno y el apoyo de los estudiantes que acuden dentro de la institución para que den un mejor servicio oportuno a las madres con hijos y pueda cumplir con un mejor rendimiento a su estudio y lleguen a dar un paso más a la vida obteniendo su título profesional. 


\section{Referencias bibliográficas.}

CDC. (2015). prebencion de embarazo. Obtenido de https://www.cdc.gov/spanish/signosvitales/larc/index.html

Fajardo Zila , Figeroa Erica,Garces Norma,. (2016). Creacion de Guarderia Universitaria.

Garcia Andres. (12 de mayo de 2018). mujeres embarazadas. Obtenido de www.el comercio.com

Garcia Andres. (12 de mayo de 2018). porcentage de mujeres embarazadas. Obtenido del comercio: www.el comercio.com

Guarderia, B. d. (12 de mayo de 2012). Obtenido de https://www.elmundo.es/elmundosalud/2012/10/30/noticias/1351626208.html

Maradiaga Cuadra Yamilia ,Weimar Flores Sherlie,. (2007). Planificacion familiar.

Noticias Redaccion Colima. (13 de diciembre de 2015). mujeres embarazadas en la adolescencia. Obtenido de mujeres embarazadas en la adolescencia: https://www.google.com.ec/search?q=mujeres+embarazadas+en+la+adolescencia\& source $=\operatorname{lnms} \&$ tbm $=i$ sch \&sa $=X \& v e d=0 a h U K E w j h s Z m z-$ rveAhWNaFAKHdTfCBEQ_AUIDigB\&biw=1366\&bih=657\#imgrc=2pvu8uvPhJ9 aOM:

ORTIZ ASAEL. (2014). Creacion Universitaria.

Universia. (2014). embarazadas a temprana edad. españa.

Yamilia, M. C. (2007). porcentages de anticonceptivos.

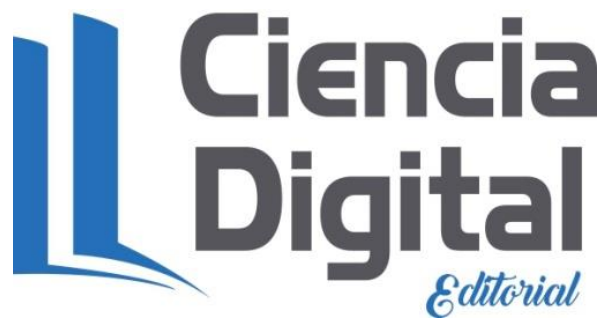


Para citar el artículo indexado.

Velasteguí López, E. (2018). Las mujeres universitaias embarazadas y el factor que incide en su avance y el rendimiento académico. ConcienciaDigital, 1(4), 34-44. https://doi.org/10.33262/concienciadigital.v1i4.905

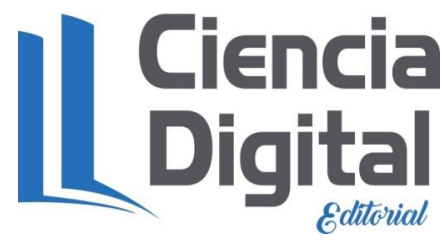

El artículo que se publica es de exclusiva responsabilidad de los autores y no necesariamente reflejan el pensamiento de la Revista Conciencia Digital.

El articulo queda en propiedad de la revista y, por tanto, su publicación parcial y/o total en otro medio tiene que ser autorizado por el director de la Revista Conciencia Digital.
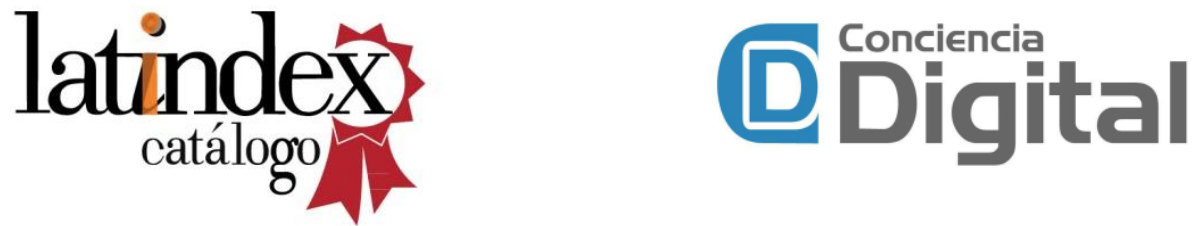